\title{
Cucurbitacin B inhibits growth and induces apoptosis through the JAK2/STAT3 and MAPK pathways in SH-SY5Y human neuroblastoma cells
}

\author{
QIAN ZHENG* ${ }^{*}$ YUNYI LIU*, WEIWEI LIU, FENGYUN MA, YI ZHOU, MINGJIE CHEN, \\ JUNLI CHANG, YUESHENG WANG, GUANGXIAO YANG and GUANGYUAN HE
}

\begin{abstract}
The Genetic Engineering International Cooperation Base of Chinese Ministry of Science and Technology, Key Laboratory of Molecular Biophysics of Chinese Ministry of Education, College of Life Science and Technology, Huazhong University of Science \& Technology (HUST), Wuhan, Hubei 430074, P.R. China
\end{abstract}

Received September 15, 2013; Accepted March 21, 2014

DOI: $10.3892 / \mathrm{mmr} .2014 .2175$

\begin{abstract}
Cucurbitacin $\mathrm{B}(\mathrm{CuB})$ is a tetracyclic triterpene that is contained in extracts from cucurbitaceous plants and has been demonstrated to have anticancer and anti-inflammatory activities. The purpose of the present study was to determine whether $\mathrm{CuB}$ exhibits anticancer effects on SH-SY5Y human neuroblastoma cells and to analyze the underlying molecular mechanism. The results demonstrated that $\mathrm{CuB}$ not only induced cell cycle arrest at the G2/M phase, but also induced apoptosis as characterized by positive Annexin V staining, downregulation of phospho-Janus kinase 2 (p-JAK2), phospho-signal transducer and activator of transcription 3 (p-STAT3), phospho-extracellular signal-regulated kinases and the activation of c-Jun N-terminal kinase and p38 mitogen activated protein kinase (MAPK). $\mathrm{CuB}$ also altered the expression of gene products that mediated cell proliferation (Cyclin B1 and cyclin-dependent kinase 1), cell survival (B-cell lymphoma 2, Bcl2-associated X protein) and increased the expression of $\mathrm{p} 53$ and $\mathrm{p} 21$. These results provide the evidence that JAK2/STAT3 and MAPKs have crucial roles in $\mathrm{CuB}$-induced growth inhibition and apoptosis in SH-SY5Y human neuroblastoma cells.
\end{abstract}

Correspondence to: Professor Guangyuan $\mathrm{He}$ or Professor Guangxiao Yang, The Genetic Engineering International Cooperation Base of Chinese Ministry of Science and Technology, Key Laboratory of Molecular Biophysics of Chinese Ministry of Education College of Life Science and Technology, Huazhong University of Science \& Technology, Luoyu Road 1037, Wuhan, Hubei 430074, P.R. China

E-mail: hegy@hust.edu.cn

E-mail:ygx@hust.edu.cn

*Contributed equally

Key words: cucurbitacin B, cell cycle arrest, apoptosis, SH-SY5Y cells

\section{Introduction}

Neuroblastoma (NB) is the most common type of extracranial solid tumor among children that arises in the peripheral sympathetic nervous system. NB typically emerges in the adrenal medulla or paraspinal ganglia during embryogenesis. NB accounts for $\sim 10 \%$ of pediatric cancer-associated mortalities, with an annual incidence of $\sim 650$ novel cases in the USA (1) and it is a malignant tumor with a low degree of differentiation. According to the degree of histopathological differentiation, neuroblastic tumors can be divided into NB, ganglioneuroblastoma and ganglioneuroma (2). The prognosis depends on the patients' age, location, region and biological characteristics of NB, but it is usually poor. In recent years, as methods of early diagnosis have improved, the survival rate of children with NB has significantly increased; however, patients with high-risk cases still have a $<40 \%$ chance of survival. Therefore, it is urgent to search for a novel adjuvant agent suitable for the treatment of NB with few side effects in order to increase the overall survival rate.

Cucurbitacins are a group of tetracyclic triterpenes isolated from Cucurbitaceae (3). Previous studies have demonstrated that they have a broad range of pharmacological effects, including anti-inflammatory $(4,5)$, anti-fertility (5), anti-viral (6) and anticancer $(4,7,8)$ activities. Chemically, cucurbitacins are highly diverse and are arbitrarily divided into 12 categories (5). Cucurbitacin $\mathrm{B}(\mathrm{CuB})$ is one of the most abundant forms of cucurbitacins and the most widely used as an anticancer agent. It has significant anti-inflammatory activity and is used traditionally to treat hepatitis (9). Studies indicate that $\mathrm{CuB}$ is capable of inhibiting the growth of a wide spectrum of malignant human cells, including myeloid leukemia, breast cancer, glioblastoma multiforme, pancreatic cancer, laryngeal cancer, melanoma and osteosarcoma cells (10-16). The anticancer mechanism of $\mathrm{CuB}$ is activated by different signaling pathways in different cancer cells. Several studies have shown that $\mathrm{CuB}$ induces apoptosis by inhibiting the Janus kinase 2 (JAK2)/signal transducer and activator of transcription 3 (STAT3) signaling pathway $(13,14)$, but others have indicated that $\mathrm{CuB}$ is a potent inhibitor of nuclear factor 
$\kappa$-light-chain-enhancer of activated B cells activation (17), and additionally exhibits anticancer effects through wingless type signaling (11).

In previous years, researchers have searched for novel effective drugs with few side effects suitable for the treatment of NB. Gheeya et al (18) used a panel of drugs in order to identify novel effective chemotherapeutics against NB, and they demonstrated that cucurbitacin I inhibits cell growth through inhibition of the STAT3 pathway. Although there is an increase in the amount of evidence indicating that $\mathrm{CuB}$ is also an inhibitor of the STAT3 pathway in several tumor cell lines $(8,13,14,19)$, whether $\mathrm{CuB}$ exhibits anticancer effects in NB cells and its exact molecular mechanism remain to be elucidated. In the present study, the effects of $\mathrm{CuB}$ on human NB SH-SY5Y cells were evaluated, and the molecular mechanisms underlying $\mathrm{CuB}$-induced apoptosis were studied.

\section{Materials and methods}

Chemicals and reagents. $\mathrm{CuB}$ at $98 \%$ purity was purchased from Must Biotechnology Co., Ltd. (Chengdu, China). MTT, dimethyl sulfoxide (DMSO), PD98059, SP600125, SB203580 and interleukin (IL)-6 were purchased from Sigma (St. Louis, MO, USA). Dulbecco's modified Eagle's medium (DMEM) and fetal bovine serum (FBS) were acquired from HyClone Laboratories (Logan, UT, USA). Propidium iodide (PI), AG490 and bicinchoninic acid (BCA) Protein Assay kit were obtained from Beyotime (Shanghai, China). An Annexin V-fluorescein isothiocyanate (FITC)/PI apoptosis detection kit was purchased from MultiSciences Biotech Co., Ltd (Hangzhou, China). The primary antibodies specific to p53 (rabbit pAb), p21 (mouse mAb), B-cell lymphoma 2 (Bcl-2; rabbit $\mathrm{pAb}$ ), Bcl2-associated $\mathrm{X}$ protein (Bax; rabbit pAb), phospho-JAK2 (Tyr 1007/1008; rabbit mAb), JAK2 (mouse mAb), phospho-STAT3 (Tyr705; mouse mAb), phospho-extracellular signal-regulated kinases (p-ERK; Thr202/Tyr204; rabbit pAb), phospho-p38 (Thr180/Tyr182; rabbit pAb) and $\beta$-actin (mouse $m A b$ ) were obtained from Santa Cruz Biotechnology (Santa Cruz, CA, USA). The phospho-c-Jun N-terminal kinase (p-JNK; Thr183/Tyr185; rabbit mAb) primary antibody was purchased from Cell Signaling Technology (Danvers, MA, USA), cyclin-dependent kinase 1 (CDK1; mouse mAb), Cyclin B1 (rabbit pAb), STAT3 (rabbit pAb), ERK (rabbit pAb), JNK1 and 2 (rabbit pAb), and p38 primary antibody (rabbit pAb) and secondary polyclonal antibodies goat anti-mouse immunoglobulin (Ig) G horseradish-peroxidase (HRP)-conjugate and goat anti-rabbit IgG HRP-conjugate were obtained from Boster Biological Technology., Ltd. (Wuhan, China).

Cell line and culture. The human NB cell line (SH-SY5Y) was obtained from the China Center for Type Culture Collection (Wuhan University, Wuhan, China). The cells were cultured in DMEM supplemented with 10\% FBS at $37^{\circ} \mathrm{C}$ in a humidified atmosphere containing $5 \% \mathrm{CO}_{2}$. All the experiments were performed one day after the cells were seeded.

Cell viability assay. The effect of $\mathrm{CuB}$ on the growth and proliferation on cancer cells was assessed by measuring the metabolic activity (MTT assay). The cells were seeded in 96-well plates at a density of $2 \times 10^{4}$ cells/well with $200 \mu 1$ culture medium per well for $24 \mathrm{~h}$. On the next day, the medium was replaced with fresh medium containing different concentrations of $\mathrm{CuB}(0-128 \mu \mathrm{M})$. Subsequent to incubation for an additional 24 and $48 \mathrm{~h}$, a total of $20 \mu \mathrm{l}$ MTT [5 mg/ml in phosphate-buffered saline (PBS)] solution was added to each well and incubated at $37^{\circ} \mathrm{C}$ for $4 \mathrm{~h}$ to metabolize the MTT into formazan. Next, the supernatant was discarded and $100 \mu \mathrm{l}$ DMSO was added to each well to terminate the reaction. The absorbance was measured at $490 \mathrm{~nm}$ using a microplate reader (Model 680; Bio-Rad, Richmond, VA, USA). Three independent experiments were performed in triplicates. The percentage of proliferation was normalized relative to the control.

Cell cycle analysis. The cell cycle parameters were analyzed using a FACScan flow cytometer (Becton Dickinson, Franklin Lakes, NJ, USA). The SH-SY5Y cells were cultured with the indicated concentrations of $\mathrm{CuB}$ for $24 \mathrm{~h}$. The cells were harvested by centrifugation, then washed with cold PBS (pH 7.4) and fixed with $70 \%$ ice-cold ethanol overnight at $-20^{\circ} \mathrm{C}$. Following fixation, the cells were harvested and rinsed once with PBS (pH 7.4) and then incubated with $500 \mu 1$ PI staining solution (50 $\mu \mathrm{g} / \mathrm{ml} \mathrm{PI,} 100 \mu \mathrm{g} / \mathrm{ml} \mathrm{RNase} \mathrm{A)} \mathrm{for} 1 \mathrm{~h}$ at room temperature. The relative numbers of cells in the $\mathrm{G} 1$, $\mathrm{S}$ and $\mathrm{G} 2 / \mathrm{M}$ phases of the cell cycle were measured.

Annexin V-FITC/PI staining. The SH-SY5Y cells were exposed to the indicated concentrations of $\mathrm{CuB}$ for $24 \mathrm{~h}$. The samples of $1-5 \times 10^{5}$ cells were harvested and rinsed twice with cold PBS ( $\mathrm{pH}$ 7.4). The cells were resuspended in $500 \mu 1$ binding buffer and stained with $5 \mu \mathrm{l}$ Annexin-FITC and $10 \mu 1$ of PI for $5 \mathrm{~min}$ in the dark at room temperature. The stained apoptotic cells were counted using a FACScan flow cytometer.

Western blot analysis. Following treatment with the indicated concentrations of $\mathrm{CuB}$, the SH-SY5Y cells were lysed and the protein concentration was determined using a BCA protein assay kit. The lysate containing $40 \mu \mathrm{g}$ of protein was subjected to SDS-PAGE. The protein was transferred to a nitrocellulose membrane, and the membrane was blocked overnight with $1 \mathrm{X}$ Tris-Buffered Saline containing $0.1 \%$ Tween-20 and 5\% skimmed milk at $4{ }^{\circ} \mathrm{C}$. Following blocking, the membrane was washed three times and incubated with the respective primary antibodies for $2 \mathrm{~h}$ at room temperature. Next, the membrane was washed three times and incubated with the diluted HRP-conjugated secondary antibody $(1: 5,000)$ for $1.5 \mathrm{~h}$ at room temperature. Subsequent to the three washes, the membrane was detected using an enhanced chemiluminescence kit (Millipore, Bedford, MA, USA).

Statistical analysis. All the values are expressed as the mean \pm standard deviation. Significant differences between the groups were determined using Student's t-test and the statistical significance was expressed as ${ }^{*} \mathrm{P}<0.05$ and ${ }^{* *} \mathrm{P}<0.01$. All the figures shown represent the results from at least three independent experiments. 

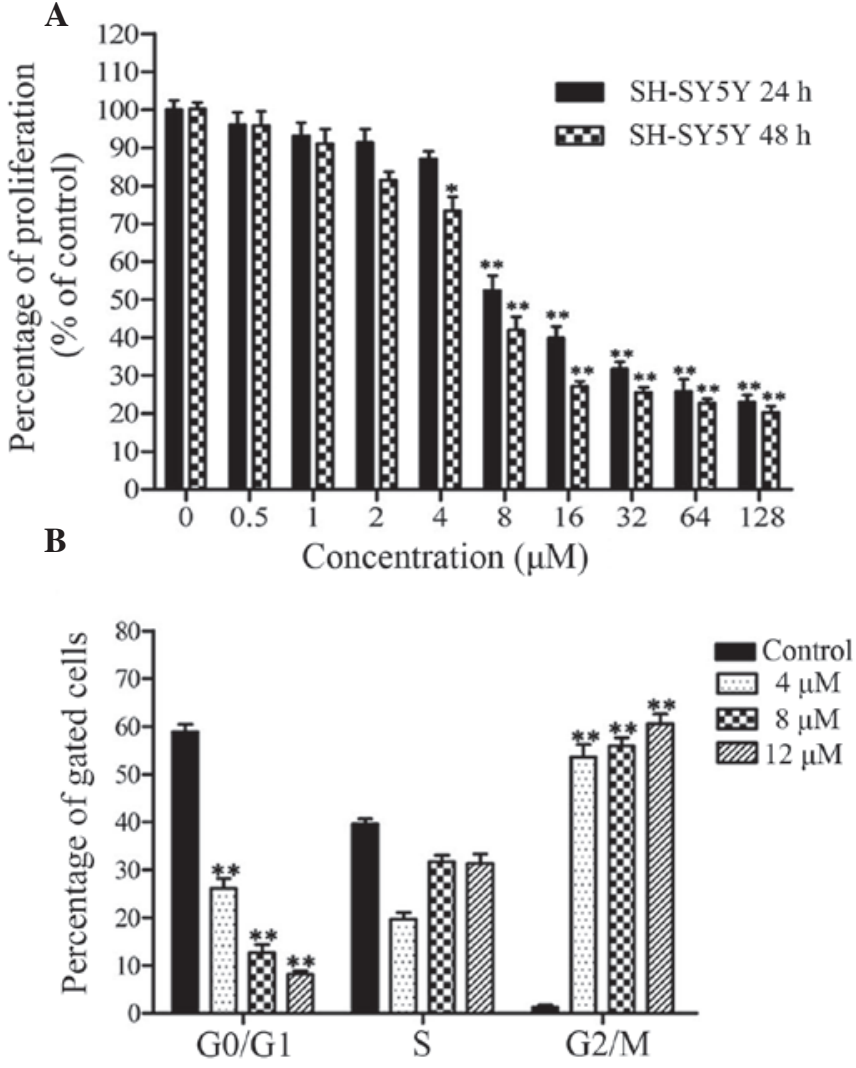

Figure 1. Effects of CuB on the proliferation of SH-SY5Y cells. (A) The SH-SY5Y cells were treated with $\mathrm{CuB}$ at the concentrations indicated for 24 and $48 \mathrm{~h}$. The viability of CuB was determined by an MTT assay. (B) The cell cycle distribution was analyzed by PI staining using flow cytometry after $\mathrm{CuB}$ treatment for $24 \mathrm{~h}$. The histograms show the cell cycle distributions in the SH-SY5Y cells. All the values are the mean \pm SD of three independent experiments. Significant differences from the control are indicated by ${ }^{*} \mathrm{P}<0.05$ and ${ }^{* *} \mathrm{P}<0.01$. CuB, cucurbitacin $\mathrm{B}$; $\mathrm{PI}$, propidium iodide; $\mathrm{SD}$, standard deviation.

\section{Results}

$\mathrm{CuB}$ inhibits proliferation of SH-SY5Y cells. In order to investigate the growth inhibition effects of CuB, the MTT assay and PI staining were performed to evaluate the cell viability and cell cycle distribution, respectively.

The viability of cancer cells treated with $\mathrm{CuB}$ was investigated by the MTT assay. CuB inhibited cell proliferation in SH-SY5Y cells in a time- and dose-dependent manner (Fig. 1A). The concentrations of 4,8 and $12 \mu \mathrm{M} \mathrm{CuB}$ were used in subsequent experiments.

Cell cycle arrest caused by $\mathrm{CuB}$ for $24 \mathrm{~h}$ was investigated by flow cytometry following PI staining. Evident changes were found in the cell cycle distributions when treated with $\mathrm{CuB}$ (Fig. 1B). The percentage of cells in the G2/M phase was increased in a dose-dependent manner. These data indicated that $\mathrm{CuB}$ treatment suppressed cell proliferation through an increased accumulation of cells in $\mathrm{G} 2 / \mathrm{M}$ phase of the cell cycle.

CuB induces early apoptosis in SH-SY5Y cells. Apoptosis in SH-SY5Y cells was detected by flow cytometry following Annexin V-FITC/PI double staining. The results revealed that the early apoptotic rate (Annexin V-FITC-positive and
A
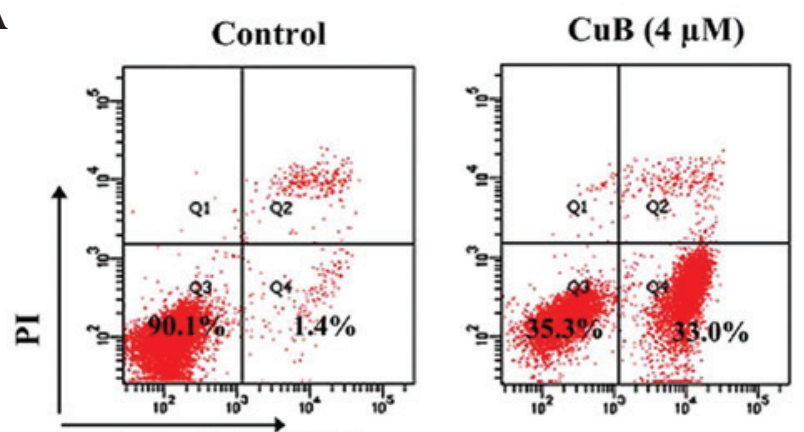

Annexin V-FITC

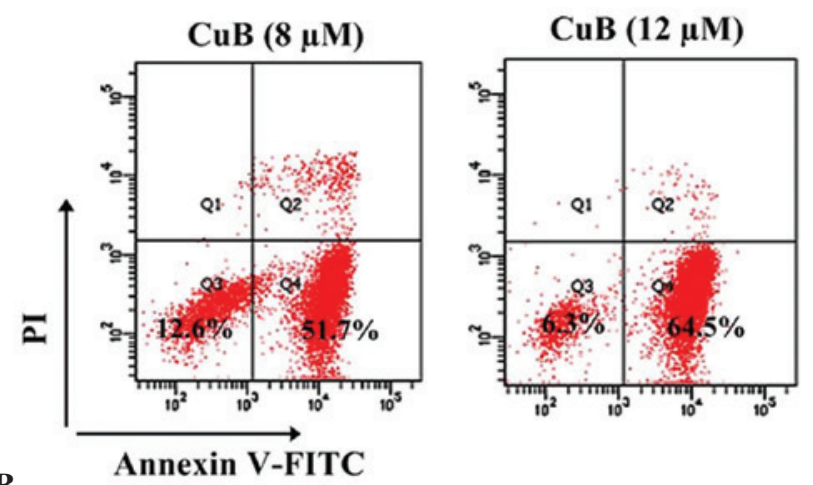

B

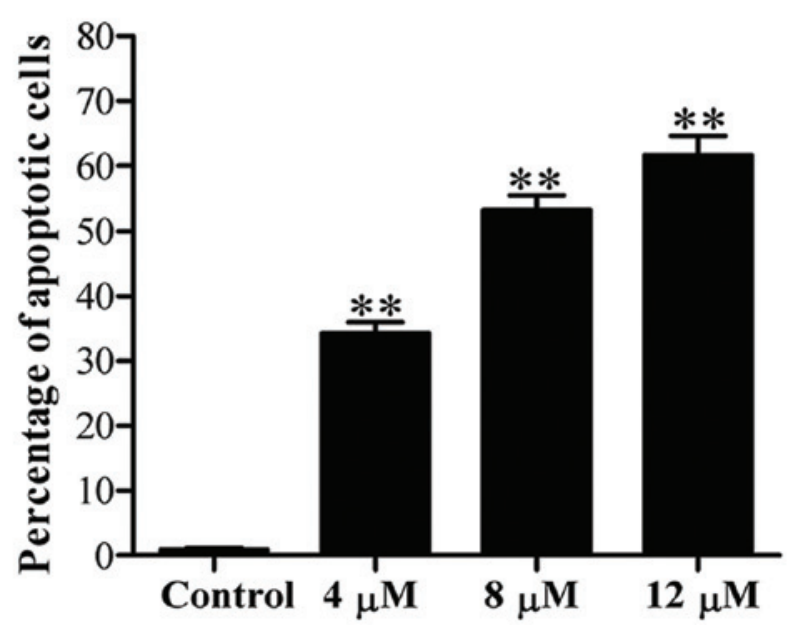

Figure 2. Effects of $\mathrm{CuB}$ on the apoptosis of SH-SY5Y cells. (A) The cells were treated with $\mathrm{CuB}$ for $24 \mathrm{~h}$, the apoptotic effect of $\mathrm{CuB}$ was assessed by flow cytometric analysis after staining with Annexin V-FITC/PI. The right bottom quadrant represents the Annexin V-stained cells (early-phase apoptotic cells). (B) Histograms showing the early apoptotic rate in SH-SY5Y cells. The values are the mean \pm SD of three independent experiments. Significant differences from the control are indicated by ${ }^{*} \mathrm{P}<0.05$ and ${ }^{* *} \mathrm{P}<0.01 . \mathrm{CuB}$, cucurbitacin B; FITC, fluorescein isothiocyanate; PI, propidium iodide; SD, standard deviation.

PI-negative cells) was significantly increased following CuB treatment for $24 \mathrm{~h}$ and the rate increased in a dose-dependent manner (Fig. 2).

$C U B$ inhibits JAK2/STAT3 signaling cascades. The phosphorylated forms of JAK2 and STAT3 were assessed by western blot analysis in SH-SY5Y cells treated with $\mathrm{CuB}$ for $24 \mathrm{~h}$. Constitutive activation of JAK2 and STAT3 were suppressed by $\mathrm{CuB}$ in a concentration-dependent manner (Fig. 3A). Next, the role of JAK2/STAT3 in CuB-induced apoptosis by a JAK2 inhibitor (AG490) and an activator of JAK2/STAT3 (IL-6) 
A

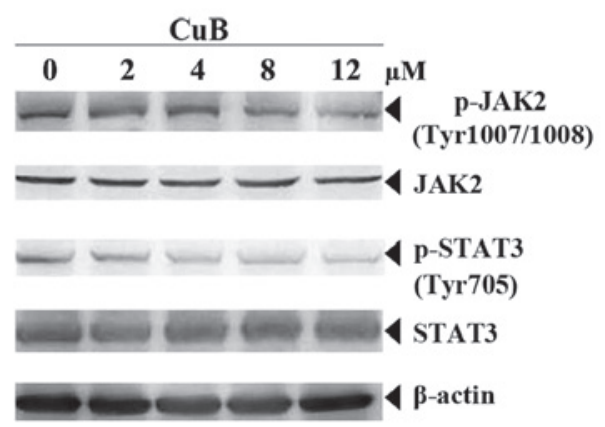

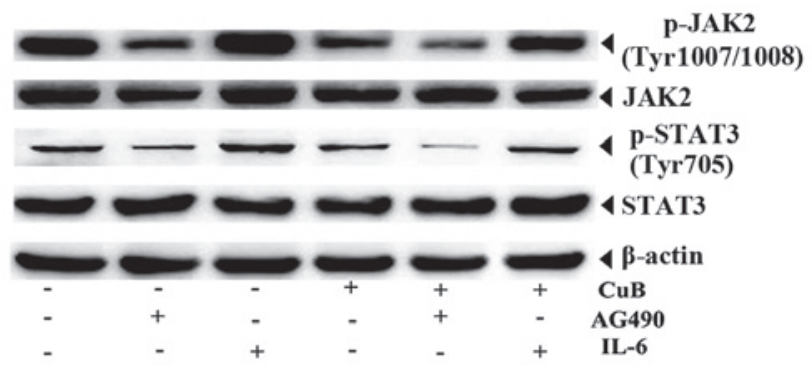

C

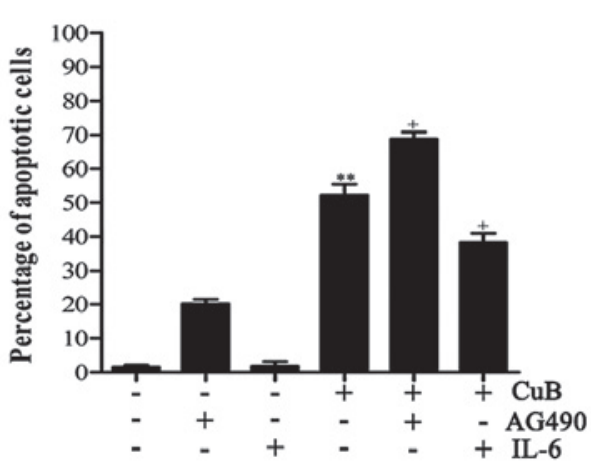

Figure 3. Effect of CuB on JAK2/STAT3 signaling cascades. (A) Cell lysates from the SH-SY5Y cells treated with CuB for $24 \mathrm{~h}$ were analyzed by western blot analysis using antibodies against p-JAK2, JAK2, p-STAT3 and STAT3, and $\beta$-actin was used as a loading control (bottom panel). (B) The cells were preincubated with or without AG490 or IL-6 for $1 \mathrm{~h}$ and further incubated in the presence or absence of $8 \mu \mathrm{M} \mathrm{CuB}$ for $24 \mathrm{~h}$. Next, equal amounts of protein were analyzed by western blot analysis. (C) The apoptotic effects of CuB, AG490 and IL-6 on SH-SY5Y cells were detected by flow cytometry. The values are provided as the mean $\pm \mathrm{SD}$ of three independent experiments. Significant differences are indicated by ${ }^{* *} \mathrm{P}<0.01$ versus control and ${ }^{\mathrm{C}} \mathrm{P}<0.05$ versus $\mathrm{CuB}$. CuB, cucurbitacin B; p-JAK2, phosphorylated Janus kinase 2; STAT3, signal transducer and activator of transcription 3; IL-6, interleukin 6; SD, standard deviation.

A

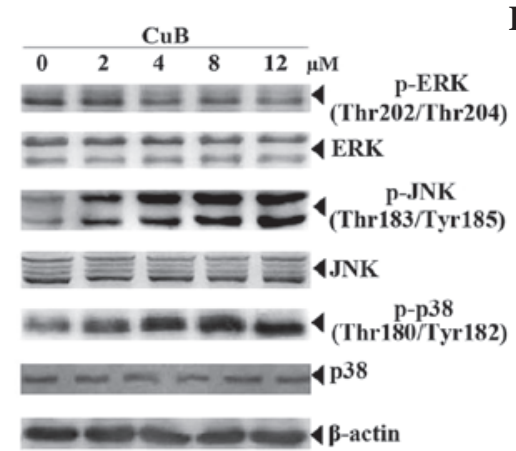

C
B

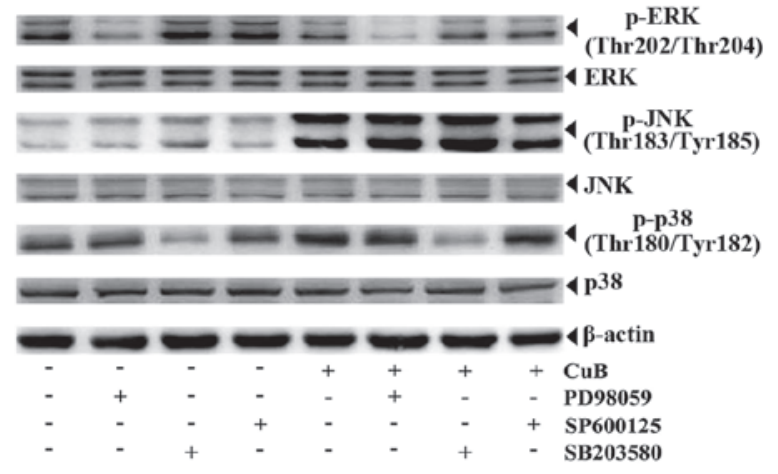

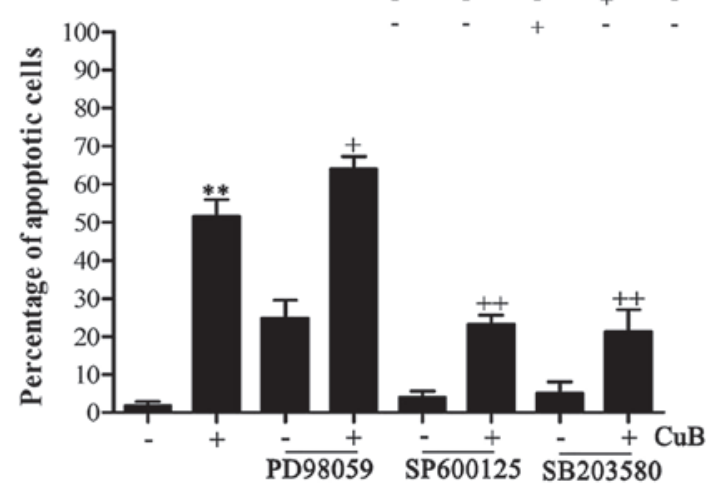

Figure 4. Effects of $\mathrm{CuB}$ on the MAPK signaling pathway. (A) The cell lysates from SH-SY5Y cells treated with CuB for $24 \mathrm{~h}$ were analyzed by western blot analysis using antibodies against p-ERK, ERK, p-JNK, JNK, p-p38 and p38, and $\beta$-actin was used as a loading control (bottom panel). (B) The cells were preincubated with or without inhibitors of signaling molecules for $1 \mathrm{~h}$ and further incubated in the presence or absence of $8 \mu \mathrm{M} \mathrm{CuB}$ for $24 \mathrm{~h}$. The concentrations of the inhibitors are as follows: PD98059, $100 \mu \mathrm{M}$; SP600125, $20 \mu \mathrm{M}$ and SB203580, $20 \mu \mathrm{M}$. Next, equal amounts of protein were analyzed by western blot analysis. (C) The apoptotic effects of the inhibitors on SH-SY5Y cells were detected by flow cytometry. The values are provided as the mean \pm SD of three independent experiments. Significant differences are indicated by ${ }^{* *} \mathrm{P}<0.01$ versus control and ${ }^{+} \mathrm{P}<0.05,{ }^{++} \mathrm{P}<0.01$ versus CuB. CuB, cucurbitacin B; MAPKs, mitogen-activated protein kinases; p-ERK, phosphorylated extracellular signal-regulated kinases; p-JNK, phosphorylated c-Jun N-terminal kinases; SD, standard deviation. 


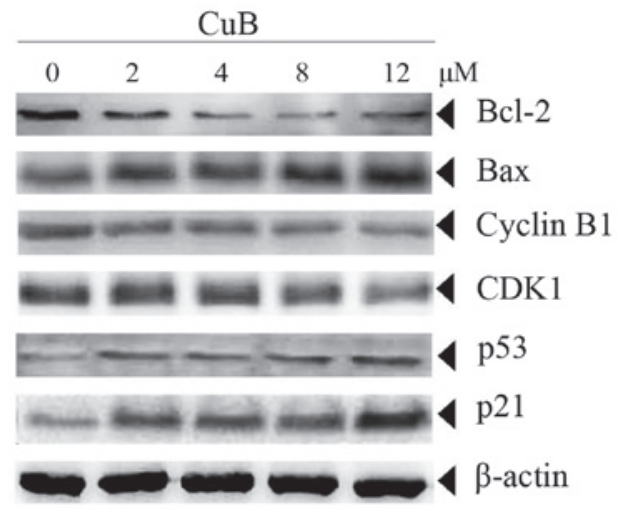

Figure 5. Effects of $\mathrm{CuB}$ on the expression of proteins involved in proliferation and apoptosis. The cells were treated with the indicated concentrations of $\mathrm{CuB}$ for $24 \mathrm{~h}$. Next, equal amounts of protein were analyzed by western blot analysis using antibodies against Bcl-2, Bax, Cyclin B1, CDK1, p53 and $\mathrm{p} 21 . \beta$-actin was used as a loading control (bottom panel). $\mathrm{CuB}$, cucurbitacin B; Bcl-2, B-cell lymphoma 2; Bax, Bcl-2-associated X protein; CDK1, cyclin-dependent kinase 1 .

was determined. Western blot analysis and flow cytometry were respectively employed to detect protein expression and apoptosis with or without AG490 or IL-6 pretreatment. As expected, the protein levels of p-JAK2 and p-STAT3 were regulated by AG490 and IL-6, while the total protein levels of JAK2 and STAT3 did not exhibit any evident change. Compared with $\mathrm{CuB}$ treatment alone, the protein levels of p-JAK2 and p-STAT3 were markedly decreased and the rate of apoptotic cells was markedly increased following the treatment of $\mathrm{CuB}$ and $\mathrm{AG} 490$. However, the alterations induced by $\mathrm{CuB}$ could be attenuated partially by IL-6 (Fig. 3B and C).

$C u B$ induces JNK and p38 MAPK activation and ERK inactivation in $\mathrm{SH}-\mathrm{SY}$ YY cells. Western blot analysis was performed to determine whether $\mathrm{CuB}$ affects the activation of MAPK cascades, including ERK, JNK and p38 MAPK in NB cells. As a result, $\mathrm{CuB}$ upregulated p-JNK and p-p38 MAPK, and downregulated p-ERK in SH-SY5Y cells (Fig. 4A). Therefore, PD98059, SB203580 and SP600125, which are specific inhibitors of ERK, p38 MAPK and JNK, respectively, were used to examine the role of the MAPK signaling pathway in CuB-treated cells. Upon pretreatment with the inhibitors, the protein levels of p-ERK, p-JNK and p-p38 MAPK were all decreased, and the decrease in p-ERK expression was significant compared with $\mathrm{CuB}$ treatment alone. ERK, JNK and p38 MAPK did not reveal evident changes (Fig. 4B). Apoptosis analysis by flow cytometry revealed that the ERK inhibitor PD98059 increased the percentage of apoptotic cells induced by $\mathrm{CuB}$, whereas the rates of apoptotic cells induced by $\mathrm{CuB}$ were significantly abrogated by the p38 MAPK and JNK inhibitors (Fig. 4C).

$C u B$ alters expression of proteins involved in proliferation and apoptosis. Bcl-2 and Bax have been implicated in apoptosis and mitochondrial dysfunction. For this reason, the effects of $\mathrm{CuB}$ on the expression of these two proteins were investigated. The data in Fig. 5 demonstrated that $\mathrm{CuB}$ downregulated the anti-apoptotic Bcl-2 and upregulated pro-apoptotic Bax in a concentration-dependent manner in SH-SY5Y cells.
In addition, cell cycle proteins linked with the $\mathrm{G} 2 / \mathrm{M}$ phase, including cyclin B1 and CDK1, were also downregulated by $\mathrm{CuB}$ in a dose-dependent manner. In multi-cellular organisms, p53 is involved in the prevention of cancer. It acts as a tumor suppressor and was reported to regulate the cell cycle through the control of the expression of cyclin-dependent kinase inhibitor p21 (20). The results indicated that p53 and p21 were also upregulated by $\mathrm{CuB}$ in a dose-dependent manner in SH-SY5Y cells (Fig. 5).

\section{Discussion}

$\mathrm{CuB}$ is known for its ability to suppress the proliferation and induce apoptosis in a wide variety of cancer cell lines, and the mechanisms of $\mathrm{CuB}$ action differ among different cancer cell lines. Identifying the molecular targets of an agent is very important in the selection of anticancer agents with few side effects on normal cells. The results of the present study indicated that $\mathrm{CuB}$ could induce cell growth inhibition by $\mathrm{G} 2 / \mathrm{M}$ phase arrest and apoptosis.

JAK/STAT3 is the major anti-apoptotic pathway for the transduction of a multitude of signals which are critical for the development and homeostasis in mammals (21). JAK activation has a significant role in cell proliferation, differentiation, migration and apoptosis $(21,22)$. Constitutive activation of STAT3 has a critical role in cell growth and survival in human solid tumor malignancies (23-25) and the upregulation of the anti-apoptotic proteins in human cancer cells $(23,26)$. JAK2/STAT3 signaling has been extensively validated as a novel molecular target for the agents against human solid tumors (27-29). In the present study, it was observed that $\mathrm{CuB}$ inhibited the JAK2/STAT3 signaling pathway by markedly downregulating p-JAK2 and p-STAT3 protein expression. It can be concluded that $\mathrm{CuB}$ may act as a JAK2/STAT3 inhibitor in SH-SY5Y cells.

MAPKs are serine-threonine protein kinases and they have a significant role in the regulation of numerous cellular processes, including cell growth and proliferation, differentiation and apoptosis $(30,31)$. MAPKs consist of growth factor-regulated ERKs, JNKs, p38 MAPK and ERK5 (32). ERK, the most widely studied MAPK cascade, has been shown to be a major participant in the regulation of cell growth and differentiation, and the activation of JNK and p38 MAPK signaling cascades generally result in apoptosis $(13,30,33)$. In the present study, $\mathrm{CuB}$ was found to activate JNK and p38 MAPK and inactivate ERK in NB cells in a concentration-dependent manner. It can be concluded that $\mathrm{CuB}$ may also act as a MAPK regulator in SH-SY5Y cells.

Bcl-2 and Bax belong to the Bcl-2 family and have a significant role in cell apoptosis. Bcl-2 and Bax exhibit antiand pro-apoptotic activities (34). The results of the present study revealed that $\mathrm{Bcl}-2$ and $\mathrm{Bax}$ were downregulated and upregulated, respectively, and further prompted apoptosis. Flow cytometric analysis revealed that $\mathrm{CuB}$ induced cell cycle arrest in the $\mathrm{G} 2 / \mathrm{M}$ phase. Cyclin $\mathrm{B} 1$ and $\mathrm{CDK} 1$ are linked to the $\mathrm{G} 2 / \mathrm{M}$ phase progress. Consistent with the results of flow cytometry, it was found that $\mathrm{CuB}$ inhibited the expression of Cyclin B1 and CDK1 in a dose-dependent manner. p53 and p21 are anticancer proteins that can induce apoptosis, inhibit Bcl-2 and cellular inhibitor of apoptosis proteins and activate 
the activities of pro-apoptosis proteins. In addition, the tumor suppressor $\mathrm{p} 53$ and its downstream target $\mathrm{p} 21$ have been shown to induce cell cycle arrest since they are potent cyclin-CDK inhibitors (35).

The present study demonstrated that human NB SH-SY5Y cells undergo apoptosis in response to treatment with $\mathrm{CuB}$, which occurs through the JAK2/STAT3 and MAPK signaling pathways, and the regulation of gene products that mediate tumor cell survival, proliferation and apoptosis. The present study also reveals that $\mathrm{CuB}$ can possibly be used as a novel potent therapeutic agent against NB. However, since all these results were obtained from in vitro experiments, in vivo studies are required in order to validate these results for the therapeutic use of this agent in humans.

\section{Acknowledgements}

This study was supported by funds from the Public Science and Technology Research Funds Projects of Ocean (no. 201005013) and the Wuhan Municipal Science and Technology Project (no. 201260523185).

\section{References}

1. Brodeur GM. Neuroblastoma: Biological insights into a clinical enigma. Nat Rev Cancer 3: 203-216, 2003.

2. Fisher JP and Tweddle DA. Neonatal neuroblastoma. Semin Fetal Neonatal Med 17: 207-215, 2012.

3. Zhang Y, Ouyang D, Xu L, Ji Y, Zha Q, Cai J and He X. Cucurbitacin $\mathrm{B}$ induces rapid depletion of the $\mathrm{G}$-actin pool through reactive oxygen species-dependent actin aggregation in melanoma cells. Acta Biochim Biophys Sin (Shanghai) 43: 556-567, 2011.

4. Jayaprakasam B, Seeram NP and Nair MG. Anticancer and antiinflammatory activities of cucurbitacins from Cucurbita andreana. Cancer Lett 189: 11-16, 2003.

5. Chen JC, Chiu MH, Nie RL, Cordell GA and Qiu SX Cucurbitacins and cucurbitane glycosides: structures and biological activities. Nat Prod Rep 22: 386-399, 2005.

6. Dzubak P, Hajduch M, Vydra D, et al. Pharmacological activities of natural triterpenoids and their therapeutic implications. Nat Prod Rep 23: 394-411, 2006.

7. Takasaki M, Konoshima T, Murata Y, et al. Anticarcinogenic activity of natural sweeteners, cucurbitane glycosides, from Momordica grosvenori. Cancer Lett 198: 37-42, 2003.

8. Liu T, Peng H, Zhang M, Deng Y and Wu Z. Cucurbitacin B, a small molecule inhibitor of the Stat 3 signaling pathway, enhances the chemosensitivity of laryngeal squamous cell carcinoma cells to cisplatin. Eur J Pharmacol 641: 15-22, 2010.

9. Promkan M, Dakeng S, Chakrabarty S, Bögler $O$ and Patmasiriwat $\mathrm{P}$. The effectiveness of cucurbitacin B in BRCA1 defective breast cancer cells. PLoS One 8: e55732, 2013.

10. Haritunians T, Gueller S, Zhang L, et al. Cucurbitacin B induces differentiation, cell cycle arrest, and actin cytoskeletal alterations in myeloid leukemia cells. Leuk Res 32: 1366-1373, 2008.

11. Dakeng S, Duangmano S, Jiratchariyakul W, U-Pratya Y, Bögler $\mathrm{O}$ and Patmasiriwat P. Inhibition of Wnt signaling by cucurbitacin B in breast cancer cells: reduction of Wnt-associated proteins and reduced translocation of galectin-3-mediated $\beta$-catenin to the nucleus. J Cell Biochem 113: 49-60, 2012.

12. Yin D, Wakimoto N, Xing H, et al. Cucurbitacin B markedly inhibits growth and rapidly affects the cytoskeleton in glioblastoma multiforme. Int J Cancer 123: 1364-1375, 2008.

13. Thoennissen NH, Iwanski GB, Doan NB, et al. Cucurbitacin B induces apoptosis by inhibition of the JAK/STAT pathway and potentiates antiproliferative effects of gemcitabine on pancreatic cancer cells. Cancer Res 69: 5876-5884, 2009.
14. Liu T, Zhang M, Zhang H, Sun C and Deng Y. Inhibitory effects of cucurbitacin B on laryngeal squamous cell carcinoma. Eur Arch Otorhinolaryngol 265: 1225-1232, 2008.

15. Oh H, Mun YJ, Im SJ, Lee SY, Song HJ, Lee HS and Woo WH. Cucurbitacins from Trichosanthes kirilowii as the inhibitory components on tyrosinase activity and melanin synthesis of B16/F10 melanoma cells. Planta Med 68: 832-833, 2002.

16. Lee DH, Thoennissen NH, Goff C, et al. Synergistic effect of low-dose cucurbitacin B and low-dose methotrexate for treatment of human osteosarcoma. Cancer Lett 306: 161-170, 2011.

17. Jin HR, Jin X, Dat NT and Lee JJ. Cucurbitacin B suppresses the transactivation activity of RelA/p65. J Cell Biochem 112: 1643-1650, 2011

18. Gheeya JS, Chen QR, Benjamin CD, et al. Screening a panel of drugs with diverse mechanisms of action yields potential therapeutic agents against neuroblastoma. Cancer Biol Ther 8: 2386-2395, 2009.

19. Zhang M, Zhang H, Sun C, Shan X, Yang X, Li-Ling J and Deng YH. Targeted constitutive activation of signal transducer and activator of transcription 3 in human hepatocellular carcinoma cells by cucurbitacin B. Cancer Chemother Pharmacol 63: 635-642, 2009.

20. Mirzayans R, Andrais B, Scott A and Murray D. New insights into p53 signaling and cancer cell response to DNA damage: implications for cancer therapy. J Biomed Biotechnol 2012: 170325,2012

21. Kiu H and Nicholson SE. Biology and significance of the JAK/STAT signalling pathways. Growth Factors 30: 88-106, 2012.

22. O'Shea JJ, Gadina M and Schreiber RD. Cytokine signaling in 2002: new surprises in the Jak/Stat pathway. Cell 109 (Suppl): S121-S131, 2002

23. Yu H and Jove R. The STATs of cancer - new molecular targets come of age. Nat Rev Cancer 4: 97-105, 2004.

24. Yu H, Pardoll D and Jove R. STATs in cancer inflammation and immunity: a leading role for STAT3. Nat Rev Cancer 9: 798-809, 2009.

25. Liu YY, Zheng Q, Fang B, et al. Germacrone induces apoptosis in human hepatoma HepG2 cells through inhibition of the JAK2/STAT3 signalling pathway. J Huazhong Univ Sci Technolog Med Sci 33: 339-345, 2013.

26. Epling-Burnette PK, Liu JH, Catlett-Falcone R, et al. Inhibition of STAT3 signaling leads to apoptosis of leukemic large granular lymphocytes and decreased Mcl-1 expression. J Clin Invest 107: 351-362, 2001.

27. Nam S, Xie J, Perkins A, et al. Novel synthetic derivatives of the natural product berbamine inhibit Jak2/Stat 3 signaling and induce apoptosis of human melanoma cells. Mol Oncol 6: 484-493, 2012.

28. Bill MA, Nicholas C, Mace TA, et al. Structurally modified curcumin analogs inhibit STAT3 phosphorylation and promote apoptosis of human renal cell carcinoma and melanoma cell lines. PLoS One 7: e40724, 2012.

29. Um HJ, Min KJ, Kim DE and Kwon TK. Withaferin A inhibits JAK/STAT3 signaling and induces apoptosis of human renal carcinoma Caki cells. Biochem Biophys Res Commun 427: 24-29, 2012.

30. Park KR, Nam D, Yun HM, et al. $\beta$-Caryophyllene oxide inhibits growth and induces apoptosis through the suppression of PI3K/AKT/mTOR/S6K1 pathways and ROS-mediated MAPKs activation. Cancer Lett 312: 178-188, 2011.

31. Scuteri A, Galimberti A, Maggioni D, et al. Role of MAPKs in platinum-induced neuronal apoptosis. Neurotoxicology 30: 312-319, 2009.

32. Boutros T, Chevet E and Metrakos P. Mitogen-activated protein (MAP) kinase/MAP kinase phosphatase regulation: roles in cell growth, death, and cancer. Pharmacol Rev 60: 261-310, 2008.

33. Ishdorj G, Johnston JB and Gibson SB. Cucurbitacin-I (JSI-124) activates the JNK/c-Jun signaling pathway independent of apoptosis and cell cycle arrest in B leukemic cells. BMC Cancer 11: 268, 2011.

34. Cory S and Adams JM: The Bcl2 family: regulators of the cellular life-or-death switch. Nat Rev Cancer 2: 647-656, 2002.

35. Fuster JJ, Sanz-González SM, Moll UM and Andrés V: Classic and novel roles of p53: prospects for anticancer therapy. Trends Mol Med 13: 192-199, 2007. 